\title{
La investigación documentológica: hacia un ajuste interdisciplinar
}

\author{
Antonio García Gutiérrez \\ Catedrático, Departamento de Comunicación \\ Universidad de Sevilla
}

\subsection{Resumen}

Reflexiones sobre el pasado, presente y futuro de la investigación documentológica (ID). Se propone el anclaje de la ID en las Ciencias Cognitivas desde una perspectiva pragmática e interdisciplinar. El objeto de la ID queda definido como la información desde el punto de vista de la recuperación, objetivo que debe guiar selectivamente los esfuerzos integración interdisciplinar. Desde esta perspectiva, se critican abordamientos reduccionistas, especialmente los que centran la ID a la simple investigación técnica desde meros presupuestos empresariales, o, en el otro extremo, los que se pierden en los vericuetos teóricos de sus propios puentes interdisciplinares, especialmente los efectuados desde la Lingüística, el Análisis del Contenido y el Análisis del Discurso. Se propone un modelo culturológicocognitivo del proceso documental en cuatro fases: lectura, transformación, representación y recuperación. Se plantea la necesidad de completar la teorización sobre el proceso documental con sendas Teorías de la Selección y del Uso de la información documental, acompañada de una sólida reflexión y depuración terminológica. Finalmente, se ofrece como línea de investigación para la próxima década la simulación de los modelos teóricos mediante sistemas expertos.

Palabras clave: Investigación. Ciencias de la Documentación. Proceso documental. Modelos Teóricos. Docencia. Planes de Estudio. Interdisciplinariedad.

\subsection{Abstract}

Reflections on the past, present and future of documentary research. Documentary research is to be approached with pragmatical aims and an interdisciplinary basis in the frame of the Cognitive Sciences. Its object is defined to be the information from the point of view of its retrieval. This aim must guide selectively any interdisciplinary approach. From this perspective, reductionist approaches are criticised: both those from a mere business and technical pers- 
pective, and those interdisciplinary ones from the Linguistics, the Content Analysis and Discourse Analysis that lose the pragmatic aims of documentary research. A careful terminological purge is, therefore, needed. The author proposes a cultural-cognitive model of the documentary process, divided into four phases: reading, transformation, representation and retrieval. This model is to be completed with a Theory of Selection and a Theory of Use of documentary information. The research task of the next decade is considered to be the simulation of the new theoretical models with the help of expert systems.

Keywords: Research. Documentary Sciences. Documentary Process. Theoretical models. Teaching. Curricula. Interdisciplinarity.

Una de las características más importantes, que determina la llamada Sociedad de la Información, es el crecimiento del volumen de información, en general, y de conocimiento científico en particular. Supuestamente, la Documentación, como disciplina académica, nace para dar respuesta al problema cuantitativo, seleccionando cualitativamente y canalizando adecuadamente los datos hacia los núcleos de usuarios pertinentes. Sin embargo, al aliarse con la Informática o la Tecnología, la Documentación corre el riesgo de estancarse como área auxiliar de aquellas, desvinculándose del ejercicio epistemológico que debe realizar con frecuencia para aferrarse a las disciplinas que le son más afines y que podríamos situar dentro de y en torno a las Ciencias Cognitivas.

En efecto, el objeto de trabajo práctico sobre el que operan los documentólogos, en sus observaciones científico-aplicadas, y los documentalistas, en sus desarrollos profesionales, es conocimiento soportado en documentos y, en función de éste, deben proponerse los modelos teóricos y montarse las metodologías. En ese sentido se define una de las personas que más ha inspirado mis trabajos, Jean Claude Gardin, al autodenominarse "epistemólogo práctico" del área arqueológica, campo científico sobre el que centra sus propuestas de métodos documentológicos de representación en sistemas expertos.

No obstante, el objeto mencionado - el conocimiento-y su información, sin transmisión no existirían avance ni conocimiento científicos, ni es propiedad de la Documentología - como ningún otro objeto es exclusivo de ninguna ciencia o investigador - ni tiene suficiente entidad como para ser adscrito de manera contundente e inmediata al campo de investigación que nos atañe. A tenor de este factor esencial, se convocó un Seminario sobre aspectos epistemológicos de la Documentación en su cruce con la Lingüística en Sao Paulo, agosto de 1991, al que asistieron profesores investigadores de las Universidades de Sao Paulo, de la Complutense de Madrid y de Sevilla, con el fin de debatir sobre la denominación,

Scire. $1: 2$ (jul.-dic. 1995). 
el alcance y los convenios interdisciplinares de nuestro objeto, al que se le adjudicó el nombre provisional de "información desde la perspectiva de su recuperación". A pesar de la conclusión coyuntural y necesaria para poder avanzar hacia el diseño de un cuadro de prioridades de investigación documentológica, la denominación siguió sacudiéndose de ciertas cargas semánticas a la vez que reclamaba algunas ausencias como la de los términos 'necesidad', 'satisfacción' o 'uso' en lo que concierne a la información científica.

En el objeto "información desde la perspectiva de la recuperación" queda mencionado un objetivo que atañe tanto a investigadores como a profesionales, y se sugiere el concepto de representación, de manera implícita, ya que es precisa la existencia de instrumentos que mediaticen los procesos de transferencia de conocimiento. Justamente, las herramientas que facilitan la representación de las ideas científicas para su recuperación y uso y la propia recuperación, son los elementos que, por ser más estudiados tal como demostraría cualquier análisis métrico, dominan el panorama de la investigación documentológica con el consiguiente empobrecimiento de otros elementos tanto o más esenciales que los citados. Y entiéndase esta crítica dentro del área de acción que considero apropiada para la Documentación puesto que me alejaría del eje central del tema si me refiero a las cuestiones de almacenamiento y difusión, identificadas con una gran parte de la Documentología durante muchos años, tal como se refleja en las obras publicadas durante las tres últimas décadas.

Centrándonos, por tanto, en el escoramiento que sufre la Documentación en sus investigaciones, debido a los importantes pero no exclusivos pesos específicos de la representación y de la recuperación, voy a situar el problema que me ocupa en un contexto superior para, a continuación, establecer unos parámetros específicos de trabajo que equilibren nuestro objeto hacia posiciones más consistentes.

La Documentación se declara, y siempre se ha declarado, disciplina pragmática (también auxiliar aunque moleste a algunos el adjetivo secundario), pues pragmático es el trabajo de los profesionales y el sentido de su investigación. Tan es así, que muchos ya hemos asumido la vía empírica como único instrumento capaz de consolidar modelos teóricos y estudios aplicados en Documentación. Ahora bien, el pragmatismo no debe significar una falta de despegue en cuanto a la visión del objeto general, ni compartimentar de tal manera las observaciones, los métodos y los resultados que, prácticamente, no sean posibles las extrapolaciones o la facultad, necesaria, de generalizar o universalizar algunos principios.

En los últimos años, ora la Documentación se ha abierto a otras disciplinas, ora otras disciplinas han hecho escala en la nuestra. Pues bien, aun siendo defensor de la investigación interdisciplinar como única vía para otorgar estatuto cien-

Scire. 1 : 2 (jul.-dic. 1995). 
tífico a la Documentación, he de expresar una gran preocupación, desprendida de la lectura de algunos libros o de la asistencia a congresos, por el extrañamiento del objeto documentológico, aún embrionario, en beneficio de otras disciplinas tradicionales y consolidadas que, por motivo de saturación en muchos casos, optan por las aplicaciones en Documentación. Así, estudios inicialmente documentológicos se transforman y consideran estudios lingüísticos, lógicos, tecnológicos u otros. Esta preocupación se incrementa si la conectamos con el hecho de la próxima institucionalización de nuestras investigaciones en Facultades de Ciencias de la Documentación. Cuando estos Centros nazcan, el objeto habrá sido observado, con toda legitimidad, desde luego, en otras instituciones universitarias, especialmente politécnicas. La respuesta a esta preocupación pasa por la urgente re-definición de objetos y objetivos de nuestras pesquisas. De esa manera tendrán sentido, en el plano investigador, esas nuevas Facultades que, por el momento, no lo tienen. Al mismo tiempo, habremos encontrado nuestro propio espacio investigador en el entramado universitario. Por ello, deben apoyarse e imitarse iniciativas como la de incluir, en el plan de estudios de la Diplomatura de Documentación de la Universidad de Zaragoza, asignaturas en torno a la organización del conocimiento y su circulación.

Pienso que la razón superior a que obedecen estos problemas es la adscripción de los estudios y de las prácticas documentales en el modelo tecnicista que impregna las ciencias sociales en nuestra época. De acuerdo con ello, la Documentación sería vista como un mero proceso industrial en el que, efectivamente, la representación del conocimiento y su recuperación informática son los polos esenciales de interés. Evidentemente, el modelo tecnicista, desentrañado por teóricos de la Comunicación y unido a las relaciones de dependencia de los países industrializados respecto a las naciones menos desarrolladas, está al servicio de las multinacionales de la información, aquellas empresas dominantes que inculcan la idea de la Cultura y del Conocimiento como Industria e Ingeniería, respectivamente.

Por el contrario, una reflexión sobre los derroteros que en España ha tomado la docencia (de acuerdo a planes de estudios mal articulados y en muchos casos sin justificación, cuando no hechos a medida de personas sin considerar estrictos criterios académicos) y la investigación (conectada totalmente a la tecnología y/o realizada sobre temas secundarios en cuanto a la urgencia que he expresado) nos debe llevar a pensar la Documentación como un proceso puramente cultural y cognitivo auxiliado por máquinas y redes. En Documentación tenemos que empezar a explicarnos cuestiones tan distintas, pero tan afines, como los efectos individuales y sociales del impacto de los nuevos media que son los instrumentos documentales apoyados en la tecnología, los procesos cognitivos presentes en la producción del conocimento científico para articular metodologías de lectura

Scire. $1: 2$ (jul.-dic. 1995). 
objetiva (como hace, por ejemplo, el análisis logicista) y los mismos procesos aplicados al documentalista como nuevo agente productor de información, en definitiva, las claves de los textos leídos y de los producidos por mediación y sus efectos, entre otras prioridades.

De esta forma, el llamamiento que he realizado en los últimos diez años a doctorandos y colegas de mi línea de investigación en cuanto a la necesidad de interdisciplinarizar la Documentación, si se me permite el neologismo, con el fin de contribuir desde ese frente a la consolidación de su propio estatuto partiendo de la convicción de que determinados conceptos y métodos útiles ya los han propuesto otras disciplinas, se vuelve espada de doble filo. Ahora la llamada es de prudencia.

Debemos dar la bienvenida a las aplicaciones de la Lógica o de la Lingüística siempre que sean estudios contributivos al proyecto documentológico, cuyo desafío es crear una Ciencia y una Teoría de las que se habla y aún están por construir. Creo que la única propiedad que podemos atribuirnos - y sobre la que tenemos derechos y serios compromisos y deberes con la comunidad académica y, especialmente, con el alumnado - es la de exponer, públicamente y con argumentos, cuál es el espacio investigador del documentólogo, hacia dónde se desarrolla y con qué otras disciplinas se vertebra.

En consecuencia, la restricción debe guiar los criterios de selección de disciplinas. Ya he visto como algunas contribuciones de la "Lingüística documental", es decir, toma de procedimientos lingüísticos y experimentación con fines documentales, se han reducido a trabajos de Lingüística aplicada, o como aplicaciones del Análisis del Discurso en nuestra disciplina no responden a los objetivos trazados ni a su espíritu pragmático.

Por ello, creo que ya disponemos de suficiente aparato teórico-conceptual ajeno como para realizar estrictos recortes dictados por nuestro objeto. La Lingüística y sus ramificaciones, los Análisis de Contenido y del Discurso (bastante explorados en la Universidad Complutense), las manipulaciones tecnológicas necesarias para experimentar en muchos casos (propias de las Politécnicas), la Psicología cognitiva (de gran arraigo en la Universidad de Zaragoza) y otras disciplinas necesarias para el dasarrollo de la Documentación nos explican, a través de sus modelos y métodos, fenómenos típicamente documentológicos. Por tanto, y con vista a esa institucionalización que nos acecha, es urgente elaborar un inventario disciplinar comentado, por autores y obras, que sirva de guía genérica a los nuevos investigadores e investigaciones en sus basamentos transdisciplinares y de orientación a otros autores desvinculados pero que, en realidad convergen con la Documentación. Asimismo, es necesario comenzar a producir tesis doctorales en esta línea de investigación en las que se explicite, ya en los prime-

Scire. $1: 2$ (jul.-dic. 1995). 
ros epígrafes sobre modelos generales y métodos, en qué perspectiva se fundamenta el investigador.

En el sentido de la anterior argumentación, he comenzado a utilizar un pequeño modelo de trabajo investigador el cual, unido a una serie de principios teóricos y prácticos, ya comienza a dar sus frutos en algunas investigaciones recientes a la vez que lo he introducido en mi propio esquema docente. Se trata de proponer los elementos del proceso documental desde una concepción más cercana a lo cultural y cognitivo que al modelo tecnicista o empresarial al tiempo que se refleja ese objetivo en la propia terminología usada. Los elementos son: lectura (percepción - captación), transformación (paso a discurso virtual), representación (paso a discurso documental mediante instrumentos traductores en los niveles semántico y gramatical) y recuperación (procesos de obtención de conocimientos que comportan otros procesos de búsqueda y uso). El modelo se abre con el elemento información, que es extradocumental, puesto que sólo lo relevante (determinado por una, esencial e inexistente, Teoría de la Selección) es aceptado en el proceso. El mismo se cierra con elementos también extradocumentológicos como el grado de satisfacción del usuario en relación con su demanda y sus necesidades reales, a la espera de una Teoría del Uso que dé al traste con todos esos conceptos sin solidez. Véase que, hasta sobre los propios términos de la denominación del objeto, y del más medular, información, es necesaria una teorización: usamos los vocablos concepto, tema, información, aleatoriamente desconociendo qué rol desempeñan, es decir, precisamos una definición clara y pragmática de todos esos significados para poder ajustar sus denominaciones.

En el empeño de consolidar cada uno de los elementos y equilibrar la influencia de la representación y de la recuperación tan patentes en la bibliografía anglosajona me he volcado, desde hace dos años, en el desarrollo de las metodologías de percepción del texto o de lectura pragmática para su simulación. En efecto, la lectura que realiza el documentalista, lejos de ser banal e ingenua, está cargada de ideología siendo el proceso perceptor cerrado y dirigido. En el caso más aséptico, debemos pensar que lee al servicio de un usuario (modelizado), pero esto es una ingenuidad. Bajo el prisma del pragmatismo, pensamos que, para que el proceso sea posible, los resultados de la lectura de textos para varios agentes lectores deben presentar una alta coincidencia, algo que no ocurre en la realidad. Sin tener en cuenta el sometimiento del documentalista y del texto al corsé del lenguaje de representación, a su vez instrumento poco ingenuo, debe pretenderse ofrecer a los documentalistas una metodología que les ayude a leer objetivamente.

Damos por hecho que la metodología parcial también será ideológica (al servicio de la institución y, por tanto, sugerida por un código) pero al menos, en el

Scire. 1 : 2 (jul.-dic. 1995). 
nivel práctico, los resultados deben alcanzar mayor semejanza. Por ello, han sido testados distintos métodos adscritos a los análisis semánticos en la Universidad de Sao Paulo sobre bibliografía científica y, en nuestro caso, sobre discurso periodístico. Así, métodos propuestos por Fillmore y Pottier, Greimas, Lasswell, Guiraud, Teun Van Dijk y otros han ofrecido algunos resultados parciales satisfactorios aunque todavía el camino es largo y complejo para conseguir la integración de los elementos positivos y hacer que funcione un método de interrogación compacto. Mucho más larga, aún, se muestra nuestra andadura si pretendemos que estos procedimientos puedan ser simulados mediante reconocimiento artificial. No obstante, ahí está trazado nuestro camino investigador para esta década.

Por esa vía las disciplinas usadas nos han prestado ayuda fundamental pero también han contribuido a la confusión cuando no a la identificación de objetos específicamente documentológicos con los de ellas mismas. Así, el modelo anteriormente expuesto pretende ser modesta y genuinamente documentológico. Pienso que trabajando en las modificaciones y en el enriquecimiento del mismo, a un nivel de teorización o de aplicación, podremos prestar el mejor servicio a una comunidad universitaria que ve en la Documentación una esperanza de futuro profesional e investigador.

Como resultado de todo lo señalado, puedo concluir con la propuesta de algunos pasos prácticos de evidente necesidad y urgente realización:

- Convocatoria de una reunión de investigadores de la Documentación desde el punto de vista de la producción de conocimiento y de sus procesos de transferencia.

- Creación de una comisión de investigación interunivestaria en Documentología teórica y práctica que establezca directrices y prioridades de investigación en nuestra área y aproximaciones, las conecte con las emprendidas en otras instituciones extranjeras y campos afines y haga propuestas para la organización del Tercer Ciclo y Doctorado de las próximas Facultades.

- Elaboración de un documento-inventario en el que se describan las principales disciplinas, autores y obras que contribuyen a la investigación documentológica con un riguroso recorte, señalándose las principales perspectivas trabajadas en España y los Centros en que tienen lugar. Cada referencia ofrecería un resumen que indicase cuál es la aportación de la obra al discurso de nuestra disciplina.

- Participación de los investigadores de la línea cognitivo-cultural de la Documentación en la redacción de los planes de estudio de las próximas Facultades de Ciencias de la Documentación para lograr introducir asigna-

Scire. $1: 2$ (jul.-dic. 1995). 
turas esenciales, y no previstas, al menos en segundo ciclo. De esta manera se lograría una correcta formación de los futuros investigadores del área.

- Organizar una base de conocimiento como punto de partida de la información científica en nuestra materia

Sevilla a 15 de noviembre de 1992.

\section{Bibliografía básica}

Allwood, Jens ; Lars, Gunnar ; Dahl, Osten. Lógica para lingüistas. Madrid : Paraninfo, $1981.203 \mathrm{p}$.

Amaro, Regina. Contribuçao da análie do discurso para à análise docuntária: o caso da documentaçao jornalistica. Sao Paulo : ECA/USP, 1991. 87 p. + anexos.

Amat, Nuria. De la información al saber. Madrid : Fundesco, 1990. 208 p.

Angenot, M. La parole pamphlétaire : contribution à la typologie des discours modernes. París : Payot, 1982.

Bardin, Laurence. Análisis de Contenido. Madrid : Akal, 1986. 183 p.

Bobes Naves, Ma Carmen. La Semiología. Madrid : Síntesis, 1989. 167 p. (col. Lingüística;12).

Cintra, A.M. Estratégias de leitura em Documentaçao. // Smit, J. (org.). Análise documentária : a análise da sintese. Brasilia : Ibict, 1987, p. 27-35.

Colle, Raymond. Tecnologías de la Información. Santiago de Chile : Pontificia Universidad Católica, 1988. 183 p.

Cunha, Isabel (org.). Análise documentária : consideraçoes teóricas e experimentaçoes. Sao Paulo : Febab, 1989. 191 p.

Cunha, Isabel. Do mito à análise documentária. Sao Paulo : Edusp, 1990. 163 p.

Cunha, Isabel ; Kobashi, N. Análise documentária e Inteligéncia artificial. Sao Paulo : ECA/USP, 1990. 25 p. + anexos (ined. mecanograf.)

Chiornii, A. I. Introducción a la teoría de búsqueda de información. La Habana : IDICT, 1975. $332 \mathrm{p}$.

Chomsky, Noam. Reflexiones sobre el lenguaje. Barcelona : Planeta/Agostini, 1985. 278 p.

Eco, U. Signo. Barcelona : Labor, 1988. 217 p.

Ellis, Davis. New Horizons in Information Retrieval. London : Library Association, 1990. $133 \mathrm{p}$.

García Gutiérrez, Antonio. Lingüística documental : aplicación a la comunicación social. Barcelona : Mitre, 1984. 279 p.

García Gutiérrez, Antonio. Estructura lingüística de la documentación: teoría y método. Murcia: Servicio de Publicaciones de la Universidad de Murcia, 1990. 166 p.

García Gutiérrez, Antonio. Suficiencia estructural y tipología de la omisión en Análisis documental. // "Documentación de las Ciencias de la Información”.13 (1990) 73-86.

García Gutiérrez, Antonio. Análisis documental del discurso periodístico. Madrid : CTD, 1992. $160 \mathrm{p}$.

Scire. $1: 2$ (jul.-dic. 1995). 
García Gutiérrez, Antonio (comp.). Lenguajes documentales en Comunicación : metodología de análisis y experimentación. Madrid : Universidad Complutense, Dept. Periodismo III/Ibercomnet, 1992.

García Gutiérrez, Antonio ; Lucas, R. Documentación automatizada en los medios informativos. Madrid : Paraninfo, 1987. 264 p.

Gardin, J. C. Les analyses de discours. Neuchâtel : Delachaux et Niestlé, 1974. 178 p.

Gardin, J. C. et al. Systèmes experts et Sciences humaines. París : Eyrolles, 1987. 269 p.

Gardin, J. C. et al. La Logique du plausible : Essais d'Epistémologie pratique en Sciences humaines. 2éme ed. París : Maison des Sciences de l'Homme, 1987. 300 p.

Greimas, A. J. Semántica estructural : Investigación metodológica. Madrid : Gredos, 1976. $398 \mathrm{p}$.

Image et Intelligence artificielle dans l'information scientifique et technique : 6 au 10 juin 1988. Bénodet (Finistére). París : Inria, 1988. 189 p.

Izquierdo, José Mª Esquemas de Lingüística documental. Barcelona : PPO, 1990. 3 v.

Joab, Michelle. Les systémes experts. // "Image et Intelligence artificielle dans l'information... o.c., p. 115-141.

Kobashi, Nair. Análise documentária: tipologias discursivas. // Cunha, Isabel (org.). Análise documentária : consideraçoes teóricas e experimentaçoes. Sao Paulo : Febab, 1989. p.31-44.

Kobashi, Nair. Análise documentária: conssideraçoes sobre um modelo lógico- semântico. // Cunha, Isabel (org.). Análise documentária : consideraçoes teóricas e experimentaçoes. Sao Paulo : Febab, 1989. p.45-57.

Krippendorf, Klaus: Metodología de análisis de contenido: teoría y práctica. Barcelona : Paidós, 1990. 279 p.

López Yepes J. (comp.). Fundamentos de Información y Documentación. $2^{\text {a }}$ ed. Madrid : Eudema, 1990. 485 p.

Martín Barbero, Jesúa. Procesos de comunicación y matrices de cultura : itinerario para salir de la razón dualista. México : Gustavo Gili/ Felafacs, 1988. 212 p.

Menon, Bruno: Indexation automatique et intelligence artificielle: quelques questions de stratégie. // Image et Intelligence artificielle dans l'information scientifique et technique : 6 au 10 juin 1988. Bénodet (Finistére). París : Inria, 1988. p.143-175.

Pêcheux, Michel. Hacia el análisis automático del discurso. Madrid : Gredos, 1978. 374 p.

Pottier, Bernard. Lingüística general. Madrid : Gredos, 1976. 426 p.

Sagredo, Félix e Izquierdo, José $\mathrm{M}^{\mathrm{a}}$ : Concepción lógico-lingüística de la Documentación. Madrid : Ibercom- Red Comnet, 1983. 440 p.

Saussure, Ferdinand de. Curso de Lingüística general. Barcelona : Planeta/ Agostini, 1985. $292 \mathrm{p}$.

Schank, R. El ordenador inteligente. Barcelona : A. Bosch, 1986. 281 p.

Schiller, Herbert. El poder informático : Imperios tecnológicos y relaciones de dependencia. Barcelona : Gustavo Gili, 1983. 225 p.

Shannon, Claude ; Weaver, Warren. Teoría matemática de la Comunicación. Madrid : Forja, 1981. 159 p.

Scire. 1 : 2 (jul.-dic. 1995). 
Smit, Joanna (org.). Análise documentária : a análise da sintese. Brasilia : Ibict, 1987. 133 p.

Tálamo, Fátima. A definiçao semântica para à elaboraçao de glossários. // Smit, Joanna (org.). Análise documentária: a análise da sintese. Brasilia: Ibict, 1987. p.87-98.

Van Dijk, Teun. Texto y contexto : semántica y pragmática del discurso. Madrid : Cátedra, 1980. $357 \mathrm{p}$.

Van Dijk, Teun. La noticia como discurso : comprensión, estructura y producción de la información. Barcelona : Paidós, 1990. 284 p.

Wormell, I. (ed.). Knowledge Engineering : expert systems and information retrieval. London : Taylor Graham, 1987. 